\title{
Water intake of cellulose materials monitored by positron annihilation lifetime spectroscopy
}

\author{
Laura Resch (1) - Anna Karner • Wolfgang Sprengel • Roland Würschum • \\ Robert Schennach
}

Received: 21 July 2021 / Accepted: 8 December 2021 / Published online: 26 December 2021

(C) The Author(s) 2021

\begin{abstract}
In this study, for the first time, the experimental technique of positron annihilation lifetime spectroscopy (PALS) has been applied to monitor in situ the microstructural changes of cellulose-based materials, i.e. paper, during water intake. For three different cellulose samples, bleached fine paper without filler, Kraft paper without filler, and a viscose fiber sheet, the mean positron lifetime $\Delta \tau_{\text {mean }}$ showed a strong increase with time in humid atmosphere, but exhibiting different trends depending on the type of sample. For all the cellulose samples investigated, the mean positron lifetime $\Delta \tau_{\text {mean }}$ shows an initial strong increase simultaneously occurring $(\mathrm{t}<10 \mathrm{~h})$ to the mass increase of the samples due to water intake. Interestingly, the variations of $\Delta \tau_{\text {mean }}$ of the viscose fiber sheet and the Kraft paper sample both show a second increase on longer timescales $(t>60 \mathrm{~h}$ in humid atmosphere) during which the mass increase of these samples has already been saturated. The results of this study show that by the means of PALS, water transport
\end{abstract}

L. Resch $(\bowtie) \cdot$ A. Karner · W. Sprengel · R. Würschum Institute of Materials Physics, Graz University of Technology, Petersgasse 16, 8010 Graz, Austria e-mail: laura.resch@alumni.tugraz.at

R. Schennach

Institute of Solid State Physics, Graz University of

Technology, Petersgasse 16, 8010 Graz, Austria

R. Schennach

CD-Laboratory for mass transport through paper, Graz

University of Technology, Graz, Austria in paper can be reliably followed over a long timespan and it is even possible to distinguish between different types of cellulose materials. The second stage increase of the mean positron lifetime after long times in humid atmosphere for the Kraft paper sample and the pure viscose sheets even suggest that not only water intake itself can be monitored but also further atomistic processes in the material are accessible.

Keywords Positron annihilation lifetime spectroscopy · Water transport · Cellulose

\section{Introduction}

Paper is made by spraying a suspension of paper fibers (1-2 weight percent of cellulose fibers) onto a sieve followed by a drying process. Therefore, paper is a porous material by nature with pores between the fibers in the $\mu \mathrm{m}$ range and pores within the fibers in the $\mathrm{nm}$ range. As can be seen by the paper making process, water plays an important role in the material. With increasing dryness the paper becomes stronger, while with increasing water content the paper loses its strength again. Both the effects of water on paper strength (Salmen and Back 1980; Jajcinovic et al. 2018; Tejado and van de Ven 2010; Neagu et al. 2005 ) and the transport of water within paper (Waldner and Hirn 2020; Krainer and Hirn 2018; Schoelkopf et al. 2000; Ye et al. 2016; Drelich et al. 2011; Xu and 
Enomae 2014; Zhou et al. 2017) have been studied for a very long time. The moisture transport phenomena in paper and the corresponding changes in the pore and fiber structure of the material are still not fully understood. However, understanding moisture transport phenomena in cellulose-based materials would be of great importance to improve their barrier properties to water vapour and hence to increase the applicability of sustainable, bio-degradable cellulose-based packaging materials. One of the main challenges in studying the transport of water in paper is still a lack in appropriate analysis methods. In this study, the method of positron annihilation lifetime spectroscopy (PALS) is used to monitor in situ the water intake and the corresponding changes in microstructure of different cellulose materials.

Positron annihilation lifetime spectroscopy is a sensitive and specific tool for the study of open volume defects, such as pores (in the sub $\mathrm{nm}$ and $\mathrm{nm}$ range (Jean et al. 2003)) and may also yield information on the chemical environment of the positron annihilation site. In positron annihilation lifetime spectroscopy, positrons from a radioactive source, e.g., ${ }^{22} \mathrm{Na}$, are implanted into the sample where they annihilate with an electron from the sample, under the emission of two $511 \mathrm{keV} \gamma$-quanta. The positron lifetime in the sample is then determined by the time difference between the detection of a $1275 \mathrm{keV} \gamma$-quant, which is generated by the radioactive decay of ${ }^{22} \mathrm{Na}$ and the detection of one $511 \mathrm{keV} \gamma$-quantum. Positrons emitted into a solid with free electrons, such as metals annihilate rapidly resulting in positron lifetime spectra with one or two lifetime components which show values typically between 100-300 ps (Hautojärvi et al. 1979). For materials with low electron density, such as cellulose, positrons implanted in the material can interact with electrons of the sample and form so called positronium atoms. Positronium (Ps) is a metastable, bound state consisting of an electron and a positron, which can exist in two spin states: Ortho-positronium (o-Ps) consists of an electron and a positron with spin states parallel to each other and a characterisitic selfannihilation lifetime of $142 \mathrm{~ns}$ in vacuum, while $p$ Ps is formed by an electron and a positron of antiparallel spin states and exhibiting a much smaller self-annihilation lifetime of $125 \mathrm{ps}$ in vacuum. Due to the formation of positronium, positron annihilation lifetime spectra of cellulose materials include three individual lifetime components, $\tau_{1}, \tau_{2}$ and $\tau_{3}$ (Cao et al. 1997; Doyle et al. 1984), where $\tau_{1}$ and $\tau_{2}$ are of the order of few 100 ps and $\tau_{3}$ is of the order of nanoseconds. The longest lifetime component $\tau_{3}$ is associated with the formation of $o$-Ps and its lifetime can be well-correlated with the size of free volume holes in a material in which the $o$-Ps atom has been formed (Schrader and Jean 1988).

In previous studies, positron annihilation lifetime spectroscopy has for example been used to study the pore sizes of different amorphous polymers (Hagiwara et al. 2000) but further also the pore size of TEMPOoxidized cellulose nanofibril films has been investigated (Fukuzumi et al. 2011). However, earlier positron lifetime measurements in similar material types were all carried out ex situ in equilibrium states and did not address questions regarding the transport of water and the connected microstructural changes in cellulose materials. In this study, positron annihilation lifetime spectroscopy is for the first time used to monitor in situ the microscopic changes of different cellulose materials during water intake by measuring the mean positron lifetime in dependence of time in humid atmosphere.

\section{Experimental}

To monitor the process of water intake and absorption in cellulose materials, the evolution of the mean positron lifetime $\tau_{\text {mean }}$ of three different cellulose materials with time was measured in humid atmosphere. The cellulose materials were a viscose fiber sheet, a bleached fine paper without filler, and a Kraft paper without filler.

Kraft paper is made out of softwood (a mixture of spruce and pine) which provides a fiber length of about 2 to $4 \mathrm{~mm}$ and a fiber diameter in the order of about 40 to $50 \mu \mathrm{m}$. The soft wood fibers are made up of cellulose, hemicellulose and lignin. The bleached fine paper is made out of hardwood (mainly eucalyptus) and has a fiber length of about 1 to $2 \mathrm{~mm}$ and also a fiber thickness in the $40 \mu \mathrm{m}$ range. During bleaching the lignin is stripped off the paper fibers leading to fibers made of cellulose and hemicellulose. The viscose fiber sheet was made from commercial viscose fibers with a flat (approximately rectangular) shape without any additives. In the viscose process hardwood is used and all hemicelluloses and lignins are 
removed leading to pure cellulose fibers. The fiber length is about $1 \mathrm{~cm}$ and the fiber diameter in the short axis is about 10 to $20 \mu \mathrm{m}$. The different structures of the three fiber types lead to different pore structures in the corresponding sheets.

The variations of the positron lifetime were monitored for each cellulose material sample during distinct states: First, the samples were measured in the 'dry' state, which refers to laboratory conditions of approximately $(50 \pm 3) \%$ relative humidity (monitored by a hygrometer) and a temperature of $20{ }^{\circ} \mathrm{C}$. This is followed by continuous positron lifetime measurements in the so-called 'wet' state of the same sample for approximately $100 \mathrm{~h}$, where the sample environment is at relative humidity of $(80 \pm 3) \%$ while the temperature is kept constant at $20{ }^{\circ} \mathrm{C}$. In a third step, the samples are then dried again (in relative humidity of less than $\left.(10 \pm 3) \%, 20{ }^{\circ} \mathrm{C}\right)$. During the whole time of these three stages the positron lifetime is measured.

To generate the above described humidity conditions in the 'dry', 'wet' and 'dried' state a sample chamber was designed, which is depicted schematically in Fig. 1. The sample chamber consists of a closed plastic box in direct connection to a sourcesample chamber on top of it. Inside the plastic box a petri-dish is placed, which is either empty, to generate conditions for the measurements in the 'dry' state (relative humidity $50 \pm 3$ )\%), or contains water ('wet' state, relative humidity $80 \pm 3$ )\%) or silica gel to dry the sample again ('dried' state, relative humidity less than $10 \pm 3) \%$ ). To allow a high fraction of positrons to interact with the sample, two long stripes of the paper samples were loosely folded 10 times to a zig-zag structure of squares of around $1 \mathrm{~cm}^{2}$ and each placed on both sides of the ${ }^{22} \mathrm{Na}$ source. The source-samplearrangement was then wrapped with a thin wire and hanged inside the sample chamber in order to allow gaseous water molecules to penetrate into the folded source-sample-arrangement from all sides. In addition, a hygrometer and a thermometer is placed inside the measurement chamber, which monitors temperature and relative humidity (not shown in Fig. 1).

For the acquisition of the positron lifetime spectra an analogue fast-fast positron lifetime spectrometer with a full width at half maximum of the time resolution of $170 \mathrm{ps}$ is used. All positron lifetime spectra of the 'dry' state of the samples contained a minimum of $1 \cdot 10^{6}$ counts, the lifetime spectra corresponding to the 'wet' and 'dried' states were recorded in time intervalls of about $10 \mathrm{~h}$ and contain a minimum of $7 \cdot 10^{5}$ counts. The evaluation of the positron lifetime spectra was done using the program PALSfit (Kirkegaard et al. 2017).

In parallel to the measurements of the positron lifetime the mass change $\Delta m$ relative to the the mass of the 'dry' sample was measured during water intake, in

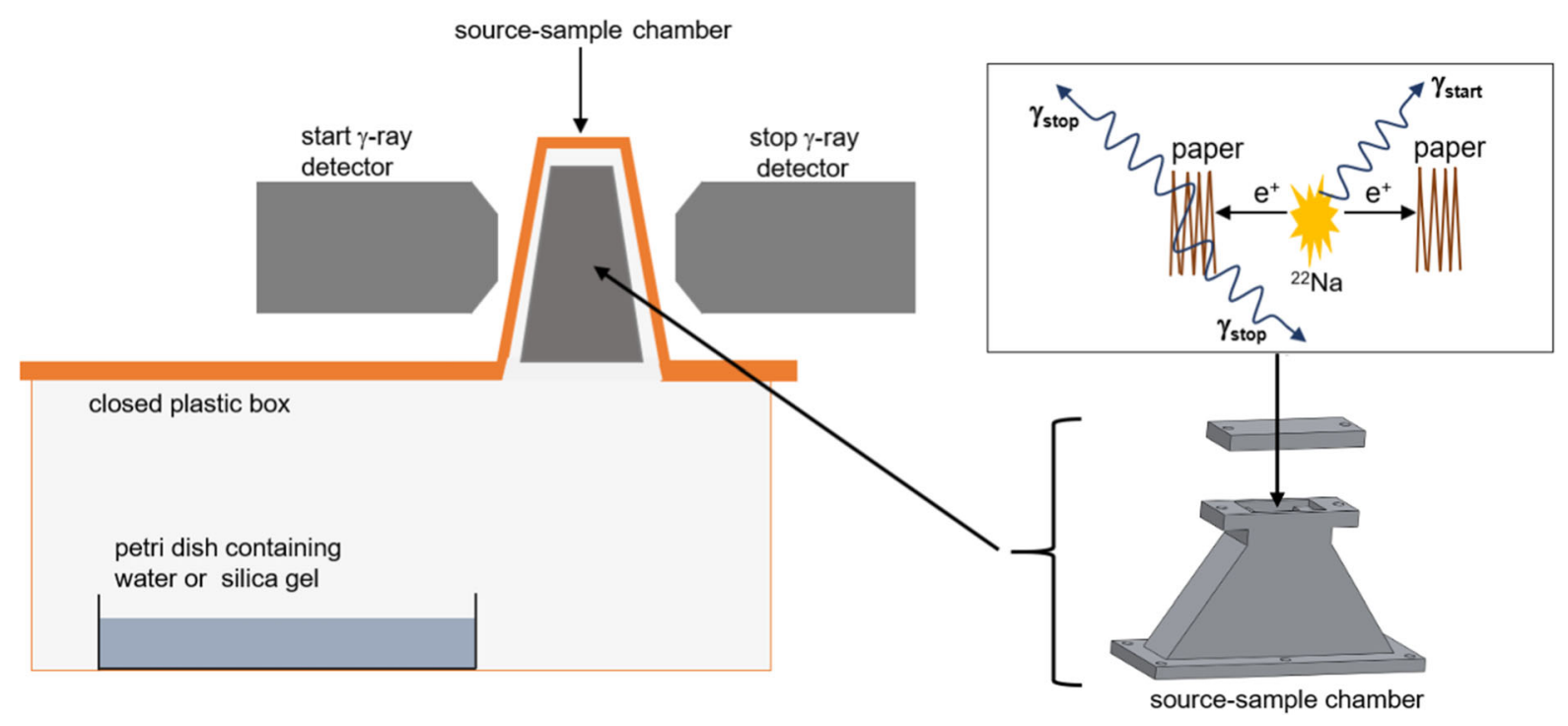

Fig. 1 Schematic sketch of the sample chamber consisting of a closed plastic box which contains a petri-dish that can be filled with water ('wet' state of sample) or silica gel ('dry' state of sample). In the cove of the box, in connection to the same atmosphere, the source-sample chamber is placed 
dependence of time in humid atmosphere ('wet' state) and during the drying process of the samples. For the measurements of $\Delta m$ sample pairs identical to those of the PALS measurements were put in a second sample chamber setup which was identical to that depicted in Fig. 1, with the exact same humidity conditions, but without the radioactive ${ }^{22} \mathrm{Na}$ source.

\section{Results and discussion}

Mass change and change of mean positron lifetime

In Fig. 2 the variation of the mean positron lifetime $\Delta \tau_{\text {mean }}$ (black dots, dashed line) with time in humid atmosphere is compared to the accompanied mass change $\Delta m$ (black crosses, dash-dotted line) for all samples investigated (viscose fiber sheet, Kraft paper without filler, bleached fine paper without filler). Note that the variations of the mean positron lifetime $\Delta \tau_{\text {mean }}$ are given as the absolute difference whereas the variations in the mass change $\Delta m$ are given as the relative change, for both with respect to the values in the 'dry' state.

For the case of bleached fine paper (see Fig. 2a), the mean positron lifetime $\Delta \tau_{\text {mean }}$ and the mass $\Delta m$ rapidly increase and reach a plateau after approximately $9 \mathrm{~h}$ in humid atmosphere. Subsequently, the values stay more or less constant, within the range of the measurement uncertainty, until the end of the measurements in the 'wet' state $(\mathrm{t} \leq 140 \mathrm{~h})$. During 'drying' ( $\mathrm{t} \geq 140 \mathrm{~h}$ ) of the fine paper sample, the mean positron lifetime $\Delta \tau_{\text {mean }}$ and the mass $\Delta m$ quickly decrease again and reach almost constant values after approximately $148 \mathrm{~h}$ (or $8 \mathrm{~h}$ from the beginning of the 'drying' state). The mass of the sample after $148 \mathrm{~h}$ reaches even lower values than the mass corresponding to the 'dry' state at the beginning of the measurement cycle, while the mean positron lifetimes obtained after $148 \mathrm{~h}$ show the same values, within the range of the errorbars, as the mean positron lifetime of the 'dry' state.

On the other hand, for the cases of Kraft paper and the viscose fiber sheet (see Fig. $2 b$ and c), the variations of the mass change $\Delta m$ and the mean positron lifetime $\Delta \tau_{\text {mean }}$ differ significantly. The mass $\Delta m$ shows a similar behavior as in the case of the bleached fine paper sample: It rapidly increases,
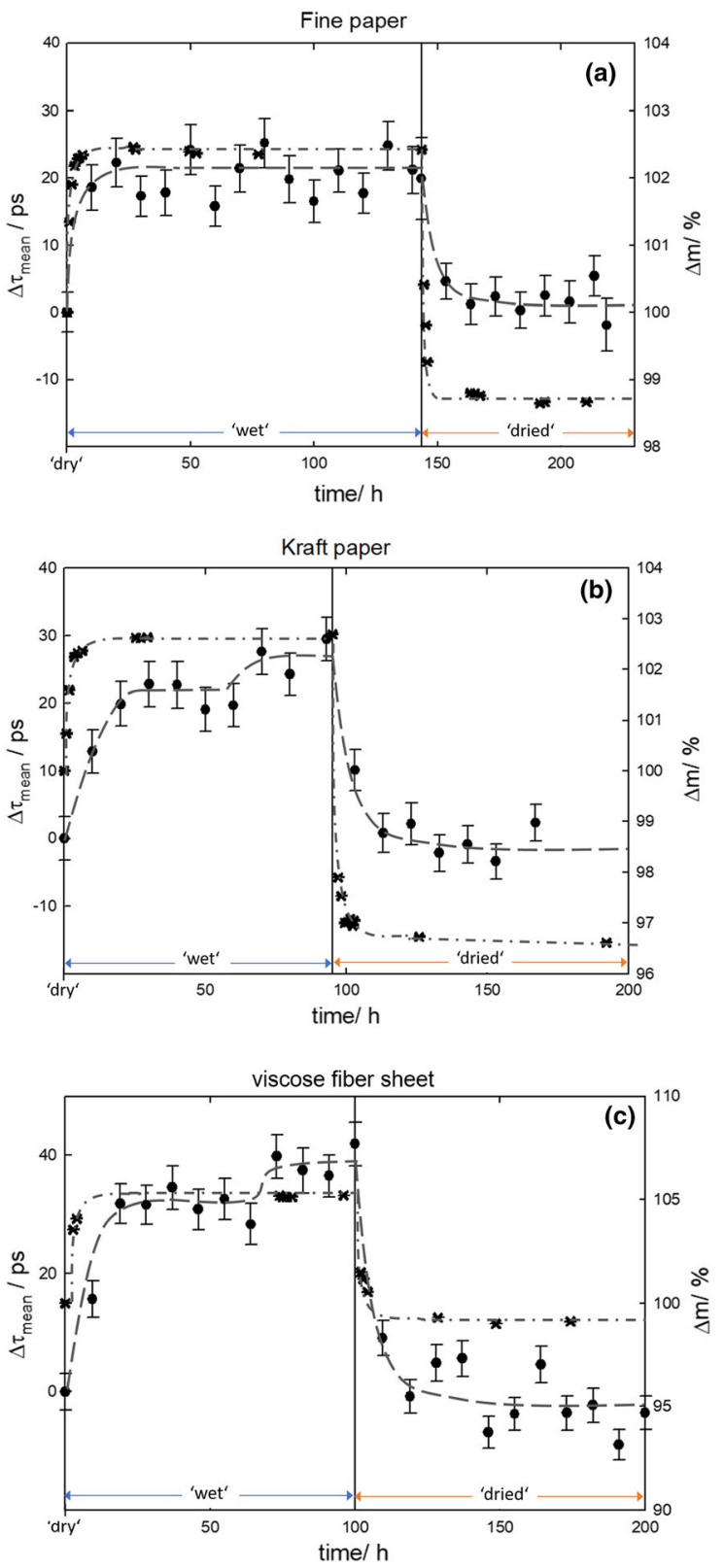

Fig. 2 Variation of the mean positron lifetime $\Delta \tau_{\text {mean }}$ (black dots, dashed line) and the mass of the samples $\Delta \mathrm{m}$ (black crosses, dash-dotted line) in dependence of time in humid atmosphere ('wet', indicated by blue arrows) and dry atmosphere ('dried', indicated by orange arrows) for a bleached fine paper without filler $\mathbf{b}$ Kraft paper without filler and $\mathbf{c}$ viscose fiber sheets. Note that the dashed and the dash-dotted lines are only guides for the eyes

reaches a maximum after approximately $9 \mathrm{~h}$ and then stays more or less constant until the end of the measurements in the 'wet' state after $95 \mathrm{~h}$ and $100 \mathrm{~h}$ 
for Kraft paper and the viscose fiber sheet, respectively. Contrarily to the bleached fine paper, the mean positron lifetime $\Delta \tau_{\text {mean }}$ increases significantly in a step-like behavior and reaches a first plateau after approximately $9 \mathrm{~h}$ in humid atmosphere. Subsequently, $\Delta \tau_{\text {mean }}$ stays more or less constant but increases again significantly after approximately 60 $\mathrm{h}$, where $\Delta \tau_{\text {mean }}$ of Kraft paper and the viscose fiber sheet reach a second plateau and stay constant until the end of the measurement in humid atmosphere. During 'drying' of the pure cellulose and Kraft paper samples, the mass $\Delta m$ and the mean positron lifetime $\Delta \tau_{\text {mean }}$ rapidly decrease and reach their minima after approximately $8 \mathrm{~h}$. It has to be noted that in the case of the cellulose fiber sheet sample, $\Delta \tau_{\text {mean }}$ shows significant fluctuations during the measurements of the 'drying' process.

For the cellulose fiber sheet sample, the overall increase of the mean positron lifetime $\Delta \tau_{\text {mean }}$ with time in humid atmosphere is higher than it is in the case of Kraft paper and bleached fine paper. This is consistent with the fact that also the mass change $\Delta m$ is highest for the case of the viscose fiber sheet. Summarizing, the variations of $\Delta \tau_{\text {mean }}$ for bleached fine paper, Kraft paper and the viscose fiber sheet show a different, reproducible behavior for each sample, and repeatedly led to different results depending on the type of paper.

\section{Lifetime components}

It is established that positron annihilation in polymeric solids results in lifetime spectra consisting of three lifetime components $\tau_{1}, \tau_{2}$ and $\tau_{3}$ attributed to the annihilation of para-positronium ( $p$-Ps), unbound positrons, and ortho-positronium (o-Ps) (Cao et al. 1997; Doyle et al. 1984). Also in the case of bleached fine paper, Kraft paper and the viscose fiber sheet all lifetime spectra could best be fitted using three individual lifetime components (after source correction) corresponding to the positron lifetimes $\tau_{1}, \tau_{2}, \tau_{3}$ and their intensities $I_{1}, I_{2}, I_{3}$, respectively. From the individual lifetime components, the mean positron lifetime $\tau_{\text {mean }}$ is calculated according to $\tau_{\text {mean }}=\tau_{1} \cdot I_{1}+\tau_{2} \cdot I_{2}+\tau_{3} \cdot I_{3}$, with $I_{1}+I_{2}+I_{3}=$ $100 \%$ (Hautojärvi et al. 1979).

The shortest lifetime component $\tau_{1}$ varied in the range from $180 \mathrm{ps}$ to $250 \mathrm{ps}$ for all samples and is attributed to $p$-Ps annihilation. The intermediate lifetime component $\tau_{2}$ varied from 350 ps to $400 \mathrm{ps}$. It can either result from fast pick-off $o$-Ps annihilation in regions of high cristallinity or result directly from the annihilation of free positrons in the medium. The third and longest lifetime component $\tau_{3}$ varied between 1500 ps and 1700 ps for all samples. Positron lifetime spectra for positron annihilation measured in water at room temperature reported by (Kotera et al. 2005; Eldrup et al. 1972) contained similar long lifetime components of approximately 1800 ps. On the other hand, positron lifetime spectra of cellulose materials (laboratory conditions, no humid atmosphere) also included such a long lifetime component $\tau_{3}$ of approximately $1700 \mathrm{ps}$ as reported in literature (Doyle et al. 1984). In the latter case, the long lifetime component was associated with the formation and annihilation of $o$-Ps within pores of the sample, and is dependent on the electron densities of the surrounding medium and on the pore size.

While the variations of lifetime components $\tau_{1}$ and $\tau_{2}$ in the present work do not show a trend during prolonged time in humid atmosphere, the variation of the longest lifetime component $\tau_{3}$ increases from approximately $1500 \mathrm{ps}$ to $1700 \mathrm{ps}$ in humid atmosphere and decreases again to values of 1400 ps$1500 \mathrm{ps}$ during drying of the samples. As a result, also the increase and decrease of the mean positron lifetime $\tau_{\text {mean }}$ in humid and 'dry' atmosphere is predominantly caused by variations of the longest lifetime component $\tau_{3}$ which is attributed to the formation of positronium. In this work, only the variations of $\tau_{\text {mean }}$ are further discussed for the above mentioned reason. Furthermore, it has to be noted that the values of the individual lifetime components underlie statistical deviations caused by short spectra acquisition times during the 'wet' and the 'drying' state.

Interpretation of lifetime variations

In general, the observed increase of the mean positron lifetime $\tau_{\text {mean }}$ is caused by variations of the $o$-Ps lifetime in the sample, and $o$-Ps, in turn, can be formed in the pores of cellulose materials or in this case by interactions with the water molecules in the samples. Hence, the observed increase of the mean positron lifetime $\Delta \tau_{\text {mean }}$ might be due to the following scenarios: (i) the adsorption of water and in connection to this an enhanced positronium formation probability, (ii) an increased pore size due to water intake, (iii) changes of 
the chemical surroundings of the water molecule due to absorption, or (iv) the penetration of water molecules into deeper pores of the sample.

As the first increase of $\Delta \tau_{\text {mean }}(\mathrm{t} \leq 20 \mathrm{~h})$ proceeds simultaneously to the change of mass $\Delta m$, it is most likely attributed to the adsorption of water molecules and an increase in pore size due to water intake. The fact that the variation of $\Delta \tau_{\text {mean }}$ shows a second increase and hence two distinct plateaus (20-60 hours, total increase of $+30 \mathrm{ps}$ and $60-100$ hours, total increase of $+10 \mathrm{ps}$ ) while the mass change $\Delta m$ saturates after approximately 10 hours is highly remarkable. It suggests that by the means of positron annihilation lifetime spectroscopy not only water intake but also further atomistic processes in connection to that can be monitored. However, even though the second increase of $\Delta \tau_{\text {mean }}$ for Kraft paper and the pure viscose sheets is reproducible and could be measured repeatedly, the interpretation of this behaviour remains speculative to this point.

One might suggest that the reason for the second increase of $\Delta \tau_{\text {mean }}$ is caused by the folded arrangement of the samples, which could induce longer times for water molecules to reach deeper layers of the sample in vicinity of the positron source. However, since the folded sample pairs were only wrapped by thin wires and hanged inside the chamber, water molecules could penetrate into deeper layers from all other directions which should minimize this effect. Further, the second plateau of $\Delta \tau_{\text {mean }}$ was repeatedly measured for Kraft paper and viscose fiber sheets but never for fine paper even though the measurement set up was identical for all samples.

On the other hand, the condensation of water after longer times in humid atmosphere could also cause a second increase of $\Delta \tau_{\text {mean }}$. But in this case, in parallel to the condensation also more water molecules should be adsorbed by the samples and the mass change $\Delta m$ should also increase, which is not the case for times in humid atmosphere longer than $20 \mathrm{~h}$.

In general, also so-called long term curl (Kajanto and Niskanen 1998; Feichtinger 1996) of the paper samples has to be considered as an explanation for the variations of the mean positron lifetime $\Delta \tau_{\text {mean }}$ after longer times in humid atmosphere. However, after the end of the 'wet' states of all samples no macroscopically visible curling of the samples was observed, and thus it can be excluded that the variations of the mean positron lifetime are related to curling.
A possible interpretation could be that the chemical environment of the water molecules change after longer times in humid atmosphere and lead to an increased $o$-Ps lifetime and hence a second plateau of $\Delta \tau_{\text {mean }}$. This process could also be connected to the penetration of water molecules into deeper pores of the sample. On the other hand, also for longer times in humid atmosphere, the variations of $\Delta \tau_{\text {mean }}$ can result from an increased pore size due to water intake, but also a combination of the above-metioned effects is possible. Furthermore, a possible explanation might also be that the Kraft paper and viscose sheet samples undergo some form of a phase transformation after $60 \mathrm{~h}$ in humid atmosphere, and that this leads to a second increase of $\Delta \tau_{\text {mean }}$.

All samples in the dried end-stage after having been exposed to a humid atmosphere exhibit a shorter $\Delta \tau_{\text {mean }}$. This fact is a clear indication that during the wet process the chain segments are rearranged, i.e., the hierarchical structure of the paper changes due to adsorption and absorption of water molecules.

\section{Summary and conclusion}

Depending on the application one might be interested in processes occurring on very short time scales when it comes, e.g., to inkjet printing, or on long time scales when one is interested, e.g., in long term curl. Positron annihilation has been demonstrated to be very sensitive on the long term effects of water adsorption on paper via the gas phase. The observed effects in terms of different adsorption and absorption properties of water on (in) paper strongly suggest that paper properties depend to a certain degree on the actual location of the water molecules in the sample. Positron annihilation is probably one of the few methods available to study the intricacies of water transport in paper. In this study we have shown that positron annihilation can follow water adsorption reliably over long time spans and we have shown that different kinds of paper lead to different results. Additional experiments with complementary methods should be done to further explore the possibilities of positron annihilation in paper research.

Acknowledgments This work was performed in the framework of the inter-university cooperation of TU Graz and Uni Graz on natural science (NAWI Graz). This work has been 
supported by the Christian Doppler Society, Austria. The financial support of the Austrian Federal Ministry for Digital and Economic Affairs and the National Foundation for Research, Technology and Development, Austria is gratefully acknowledged.

Funding Open access funding provided by Graz University of Technology. Financial support for this study was provided by the CD-Laboratory for mass transport through paper.

Availability of data and material The data that support the findings of this study are available from the corresponding author upon reasonable request.

Code availability Not applicable.

\section{Declarations}

Conflict of interest The authors declare that they have no conflict of interest.

Open Access This article is licensed under a Creative Commons Attribution 4.0 International License, which permits use, sharing, adaptation, distribution and reproduction in any medium or format, as long as you give appropriate credit to the original author(s) and the source, provide a link to the Creative Commons licence, and indicate if changes were made. The images or other third party material in this article are included in the article's Creative Commons licence, unless indicated otherwise in a credit line to the material. If material is not included in the article's Creative Commons licence and your intended use is not permitted by statutory regulation or exceeds the permitted use, you will need to obtain permission directly from the copyright holder. To view a copy of this licence, visit http://creativecommons.org/licenses/by/4.0/.

\section{References}

Cao H, Yuan J, Jean YC, Pekarovicova A, Venditti R (1997) Microstructure of cellulose studied by positron annihilation lifetime spectroscopy. Mater Sci Forum Trans Tech Publ 255:290-292

Doyle S, Malhotra BD, Peacock N, Pethrick RA (1984) Positron annihilation and $\mathrm{x}$-ray diffraction studies of cellulose and some derivatives. Brit Polym J 16(1):15-20

Drelich J, Chibowski E, Meng DD, Terpilowski K (2011) Hydrophilic and superhydrophilic surfaces and materials. Soft Matter 7(21):9804-9828

Eldrup M, Mogensen O, Trumpy G (1972) Positron lifetimes in pure and doped ice and in water. J Chem Phys 57(1):495-504

Feichtinger M (1996) Über die Auswirkung der Faserorientierung in Papierblättern. $\mathrm{PhD}$ thesis, Graz University of Technology

Fukuzumi H, Saito T, Iwamoto S, Kumamoto Y, Ohdaira T, Suzuki R, Isogai A (2011) Pore size determination of tempo-oxidized cellulose nanofibril films by positron annihilation lifetime spectroscopy. Biomacromolecules 12(11):4057-4062

Hagiwara K, Ougizawa T, Inoue T, Hirata K, Kobayashi Y (2000) Studies on the free volume and the volume expansion behavior of amorphous polymers. Radiat Phys Chem 58(5-6):525-530

Hautojärvi P, Dupasquier A, Manninen M (1979) Positrons in solids, vol 12. Springer, Berlin

Jajcinovic M, Fischer WJ, Mautner A, Bauer W, Hirn U (2018) Influence of relative humidity on the strength of hardwood and softwood pulp fibres and fibre to fibre joints. Cellulose 25(4):2681-2690

Jean JY, Mallon PE, Schrader DM (2003) Principles and applications of positron and positronium chemistry. World Scientific, Singapore

Kajanto I, Niskanen K (1998) Dimensional stability - curl. Paper Physics of Papermaking Science and Technology. In: Niskanen K (ed) published by F Oy. Finlande, Helsinki, pp 239-251

Kirkegaard P, Olsen JV, Eldrup MM (2017) Palsfit3: a software package for analysing positron lifetime spectra. DTU Library

Kotera K, Saito T, Yamanaka T (2005) Measurement of positron lifetime to probe the mixed molecular states of liquid water. Phys Lett A 345(1-3):184-190

Krainer S, Hirn U (2018) Short timescale wetting and penetration on paper. In: InterPore: InterPore2018 New Orleans

Neagu RC, Gamstedt EK, Lindström M (2005) Influence of wood-fibre hygroexpansion on the dimensional instability of fibre mats and composites. Compos Part A-Appl S 36(6):772-788

Salmen NL, Back EL (1980) Moisture-dependent thermal softening of paper, evaluated by its elastic modulus. Tappi 63(6):117-120

Schoelkopf J, Ridgway CJ, Gane PA, Matthews GP, Spielmann DC (2000) Measurement and network modeling of liquid permeation into compacted mineral blocks. J Colloid Interf Sci 227(1):119-131

Schrader DM, Jean YC (1988) Positron and positronium chemistry

Tejado A, van de Ven TG (2010) Why does paper get stronger as it dries? Mater Today 13(9):42-49

Waldner C, Hirn U (2020) Ultrasonic liquid penetration measurement in thin sheets-physical mechanisms and interpretation. Materials 13(12):2754

Xu Y, Enomae T (2014) Paper substrate modification for rapid capillary flow in microfluidic paper-based analytical devices. Rsc Adv 4(25):12867-12872

Ye Z, Li S, Wang C, Shen R, Wen W (2016) Capillary flow control in nanochannels via hybrid surface. RSC Adv 6(4):2774-2777

Zhou H, Chang R, Reichmanis E, Song Y (2017) Wetting of inkjet polymer droplets on porous alumina substrates. Langmuir 33(1):130-137

Publisher's Note Springer Nature remains neutral with regard to jurisdictional claims in published maps and institutional affiliations. 